\title{
The complexity of human infected AIV H5N6 isolated from China
}

\author{
Zhijie Zhang ${ }^{1,2}$, Rui Li ${ }^{1,2}$, Lufang Jiang ${ }^{2,3}$, Chenglong Xiong: ${ }^{2,3,5^{*}}$, Yue Chen ${ }^{4}$, Genming Zhao ${ }^{1,2}$ and Qingwu Jiang ${ }^{2}$
}

\begin{abstract}
Background: Novel avian influenza viruses (AIVs) of H7N9, H10N8, and H5N6 are currently circulating in China's poultry flocks, occasionally infecting human and other mammals. Human infected AIV H5N6 in China during 2014-2015 is believed to be a triple reassortant originated from H6N6 and two clades of H5 viruses. The current report suggests that its reassortment history is more complicated.

Methods: Genomes of human infected isolates of AIV H5N6 were searched from the NCBI Influenza Virus Sequence Database and the Global Initiative on Sharing Avian Influenza Data. Sequences shared high identities with each segment of their genomes were obtained through the Basic Local Alignment Search Tool. Alignments were done by mafft-7.037-win32 program; 8 large-scale and then 8 gradually converged phylogenetic trees were constructed by using MEGA5.1/5.2/6.0 Software.

Results: The events that each segment of the genomes of human infected AlV H5N6 isolates circulated in China had evolved into its current status might have happened before 2013, and so were they then reassorted into the epidemic AlV H5N6. A/Guangzhou/39715/2014(H5N6) and A/Sichuan/26221/2014(H5N6) had their six internal segments (PB2, PB1, PA, NP, NEP, and M) in common, and were reassorted from AIVs H5N1 in the same period and same region as that of HA, while A/Yunnan/0127/2015(H5N6) derived its six internal segments from AIV H9N2 that has been prevalent in Eastern China since 2008.
\end{abstract}

Conclusions: AIV H5N6 isolates established from both human and poultry in China during 2014-2015 were heterogeneous; both AIVs H5N1 and H9N2 were involved in the reassortment of AIV H5N6 in China.

Keywords: H5N6, Reassortment, H9N2, H5N1

\section{Background}

Avian influenza viruses (AIVs) pose significant risks to public health; novel reassortants of AIVs H7N9, H10N8, and $\mathrm{H} 5 \mathrm{~N} 6$ are currently circulating in China's poultry flocks, occasionally infecting human and other mammals [1-3]. Since a man in Sichuan province died of AIV H5N6 infection on May 6, 2014, which is the first known case of human infection of this flu subtype in the world [4], 9 confirmed human spillover infections of AIV H5N6 had been reported sporadically in China [5]. There are likely a lot more undetected H5N6 flu cases with no severe symptoms, especially in the children

\footnotetext{
* Correspondence: xiongchenglong@fudan.edu.cn

${ }^{2}$ Key Laboratory of Public Health Safety, Ministry of Education, Fudan

University, Shanghai, People's Republic of China

${ }^{3}$ Department of Public Health Microbiology, School of Public Health, Fudan

University, Shanghai, People's Republic of China

Full list of author information is available at the end of the article
}

population [6]. To reduce the threat of human infections with novel or enzootic AIV subtypes, there is an urgent need to determine the factors, which contribute to the emergence of novel AIVs. We explored the origin of human infected AIV H5N6 through its reassortment history.

\section{Methods}

Genomes of human infected isolates of AIV H5N6 were collected from the NCBI Influenza Virus Sequence Database (http://www.ncbi.nlm.nih.gov/genomes/FLU/ aboutdatabase.html) and the Global Initiative on Sharing Avian Influenza Data (GISAID) database (http://platform. gisaid.org/epi3/frontend) on December 18, 2015. Sequences shared high identities with each segment of the genomes of these AIV H5N6 isolates were obtained through the Basic Local Alignment Search Tool (BLAST); 
the parameter of max target sequences was set as 1000 . The acquired matrices were merged into 8 new ones according to their coding proteins, and the repeated sequences in each of them were removed. Alignments were done by mafft-7.037-win32 program. We first applied the Neighbor-Joining statistical method, kimura 2-parameter model, and bootstrap test with 500 replicates to construct 8 large-scale phylogenetic trees by using MEGA 5.1/5.2/ 6.0 Software. Sequences that shared high pairwise identities and sited within the same branch were remained only the earliest one unless they were isolated from different countries or regions, since they might be the same strain obtained from different host individuals. After convergence, 8 relatively small matrices were used for further analyses; and then, the Jmodeltest 2 Program was used to determine the optimum nucleotide institution model to construct accuracy phylogenetic trees, where the maximum likelihood statistical method and bootstrap test with 1000 replicates were applied. When analyzing internal 6 segments (PB2, PB1, PA, NP, NEP, and M), PanfluH1N12009 was served as the out-group; Novel AIVs H7N9 and H10N8 emerged in China during 2013-2014 also were discussed in this study.

\section{Results}

Three genomes for human infected AIVs H5N6, A/ Sichuan/26221/2014(H5N6), A/Yunnan/0127/2015(H5N6), and A/Guangzhou/39715/2014(H5N6) were obtained from the NCBI Flu Database and GISAID. And then, 8 segments, 24 matrices, and 24000 sequences were obtained through the BLAST. After removing repeated sequences, the 8 merged large-scale matrices included 2 053, 2 037, 2 029, 1 075, 2 084, 1 040, 2 146, 2062 sequences according to the segments PB2, PB1, PA, HA, NP, NA, M, NEP, respectively (Additional file 1: 1a-8a, and Additional file 2: 1b-8b). After convergence, 8 relatively small matrices consisted of $51,51,51,28,51,35,51$, 51 sequences were obtained respectively; GTR + G + I was determined to be the optimum nucleotide institution model to construct accuracy phylogenetic trees.

The data showed that each segment of the genomes of human infected isolates of AIV H5N6 circulated in China could have reached its current evolutionary status before 2013, and was then reassorted into the epidemic AIVs H5N6, which had infected poultries in China and South and Southeast Asia for a period of time before sporadical spillover infection to human beings. For HA, the three isolates of human derived AIV H5N6 might have AIV H5N1, which had circulated in China, and South and Southeast Asia since 2005 [e.g., A/Guangxi/1/2005(H5N1) or A/ duck/Vietnam/215/2005(H5N1)], served as their remote ancestor. So far, the descendants of this AIV H5N1, including AIVs H5N1, H5N2, H5N6, and H5N8, have been prevalent in Eastern China and Southeast Asia such as
Vietnam, Bangladesh, Laos, India, Nepal and others. For A/Yunnan/0127/2015(H5N6) and A/Guangzhou/39715/ 2014(H5N6), they probably descended from the same closer ancestor such as A/goose/Shandong/k1201/ 2009(H5N1) from Eastern China, while the closer ancestor of A/Sichuan/26221/2014(H5N6) is A/duck/Eastern China/1111/2011(H5N2) (Fig. 1a).

The donor of NA for AIV H5N6 was AIV H6N6 that emerged before 2004. This H6N6 was frequently isolated in the coastal areas of South and Southeast China and evolved into two clades during 2004-2007; one clade [A/duck/Shantou/6233/2004(H6N6)] was reassorted into A/Yunnan/ 0127/2015(H5N6) and A/Guangzhou/39715/2014(H5N6) and the other clade [A/duck/Fujian/3242/2007(H6N6)] into A/Sichuan/26221/2014(H5N6) (Fig. 1b).

Six internal segments have more complex reassortment characteristics. A/Guangzhou/39715/2014(H5N6) and A/Sichuan/26221/2014(H5N6) have a similar origin from AIV H5N1 in the same period and region as that of HA, but their closer ancestor is A/wild duck/Fujian/ $1 / 2011$ (H5N1), which reassorted into the two isolates of AIV H5N6 around 2013, causing an epidemic in the poultry of China, and South and Southeast Asia. While the six internal segments of A/Yunnan/0127/ 2015(H5N6) is reassorted from AIV H9N2 that had been prevalent in Eastern China since 2008, named as A/ chicken/Hebei/DF/2008(H9N2)-like-AIVs; this process was also completed as far back as 2013, but the endemic regions was only limited to the mainland China (Figs. 2 and 3, and Additional file 3: 2c, 3c, 7c, and $8 \mathrm{c}$ ).

It is worth mentioning that the internal six segments for human infected AIVs H7N9 and H10N8 emerged in China during 2013-2014 were also originated from AIV H9N2. Reassortment likely happened between AIVs $\mathrm{H} 5 \mathrm{~N} 1$ and $\mathrm{H} 9 \mathrm{~N} 2$ also, and for example, the internal six segments of A/chicken/Fujian/FZ02/2011(H9N2) were similar with AIV H5N1 more than AIV H9N2 in the same period.

\section{Discussion}

Since the first case of human infection with AIV H5N6 occurred in China in 2014, its origin has been an important topic. It is believed that the virus is a triple reassortant avian influenza virus containing an HA from clade 2.3.4.4 $\mathrm{H} 5$ viruses, a NA from H6N6 viruses, and 6 internal genes from clade 2.3.2.1 H5 viruses [7-9]. Our study however, found that concerning HA, NA, or 6 internal genes, human infected isolates of AIV H5N6 in China had other origins, and at least one strain of them had derived its 6 internal genes from AIV H9N2, which had circulated in Eastern China for a very long time. It is similar to AIVs H7N9 and H10N8, which emerged in China shortly before [1, 2]. Therefore, 


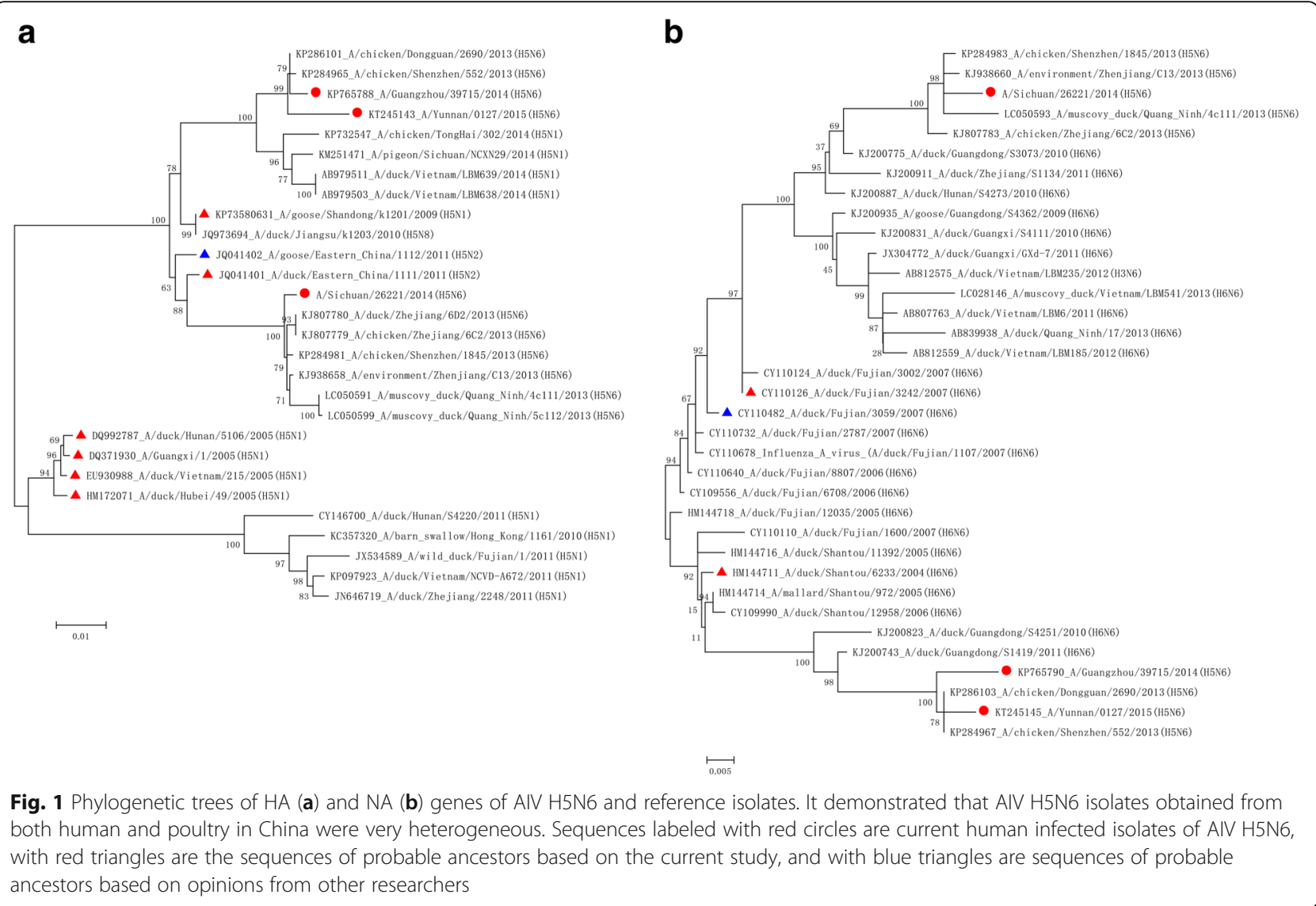

human infected AIV H5N6 in China during 20142016 is likely to have more complicated origin than it is generally believed.

As a segmented negative-strand RNA virus, influenza virus is characterized by a high frequency of reassortment. Reassortment can only occur among viruses, which replicate within the same cells. The prerequisite for reassortment is an individual host that is simultaneously infected with multiple divergent virus strains $[10,11]$.

Some regions of China have suitable conditions for the emergence of novel influenza viruses, for example, the

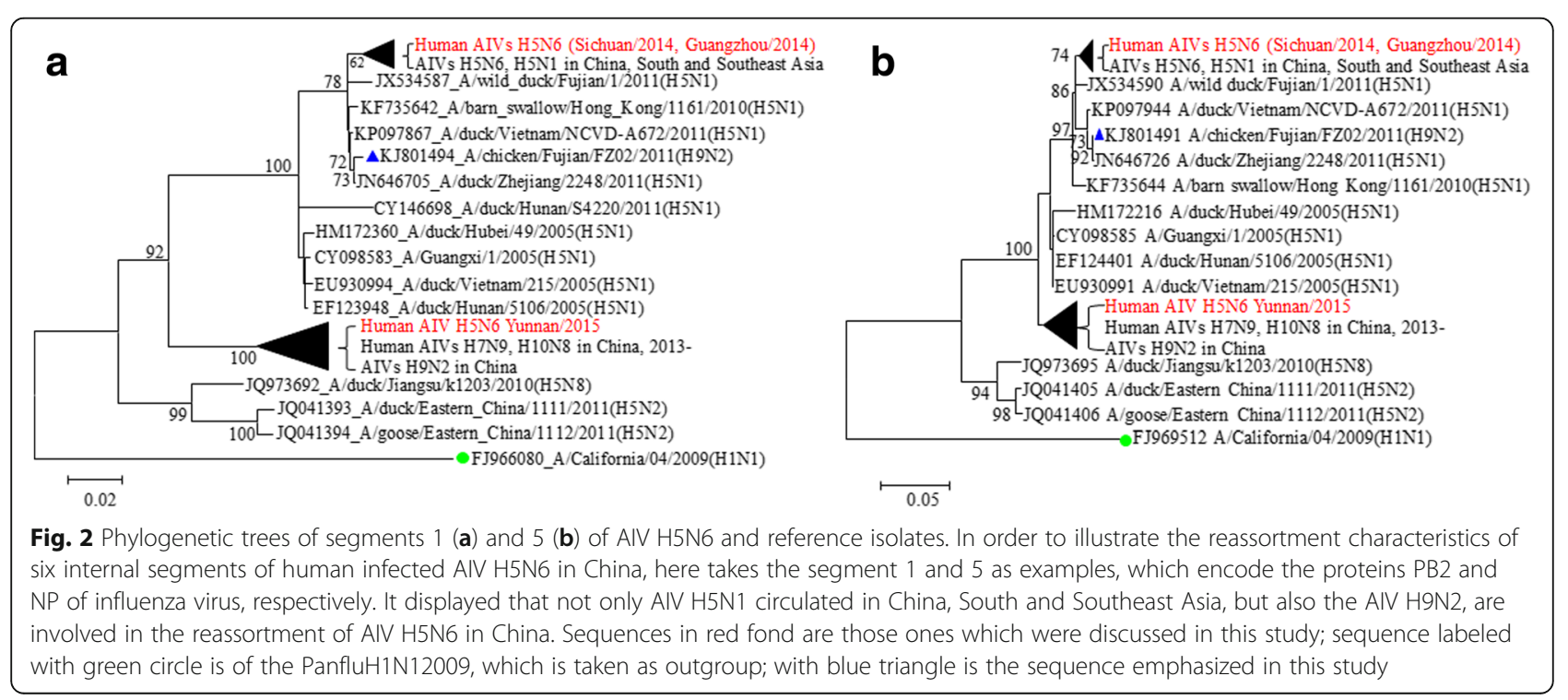




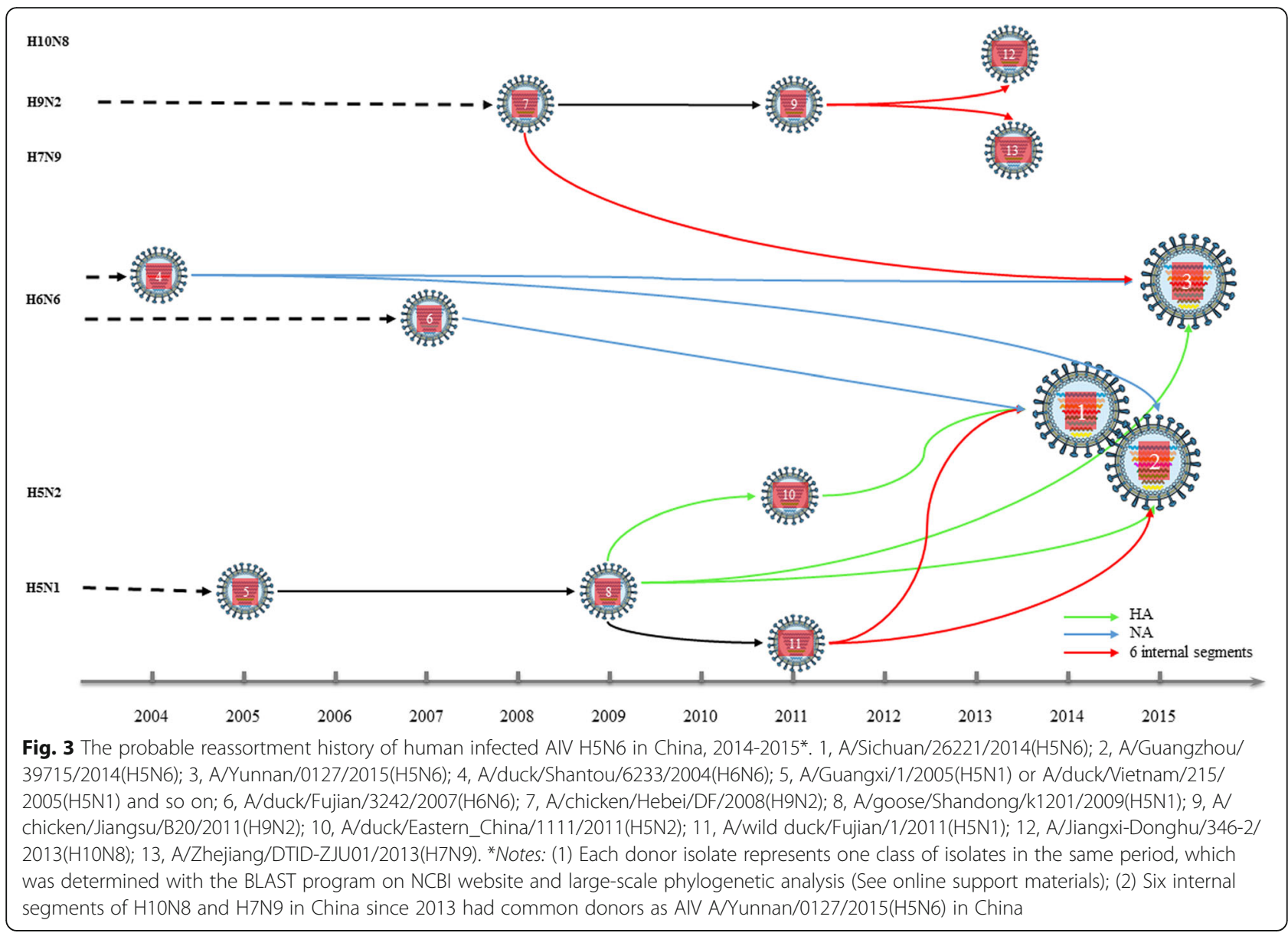

first H9N2 low-pathogenicity avian influenza virus (LPAIV) and H5N1 high-pathogenicity avian influenza virus (HPAIV) were found in Guangdong province of Eastern China in 1994 and 1996, respectively [12, 13]. Poultry farms, unvaccinated or vaccinated, had high carrying rates of AIVs $\mathrm{H} 9 \mathrm{~N} 2$ or $\mathrm{H} 5 \mathrm{~N} 1$ and often caused sporadic outbreaks [14-16]. In Eastern and Southern China, the perennial positive rate of antibody against $\mathrm{H} 9$ fluctuated between 5.3 and $12.8 \%$, and the rate of AIV H9N2 isolating could reach as high as $9 \%$ in poultry. However, there has been no obvious epidemic with mass poultry deaths $[17,18]$. High prevalence of AIVs H9N2 and $\mathrm{H} 5 \mathrm{~N} 1$ infections in poultry could result in the simultaneous infection to an individual host with multiple divergent AIVs, and then novel AIVs could occurred through reassortment. Indeed, HPAIVs strains of H5N1, H5N2, H5N6, and H5N8 have been found in China since 2000 and widely circulate currently. They occasionally cause human infections, including at least six subtypes of AIVs (H5N1, H6N1, H7N9, H9N2, H10N8, and H5N6) [16, 19, 20].

All this implies that HA5 has not only a complex evolutionary ecology, but also has a high frequency of reassortment. Understanding of the origin of novel AIVs will help targeting for early detection and containment of these viruses. Furthermore, on the HA1/HA2 cleavage site AIV H5N6 has a motif of 321-PLREKRR/KR*GLF332 with a typical feature of high pathogenicity $[7,9$, 21-24]; it means that both infection with poultries and spillover to human sporadically, such as AIV H5N6 emerged in China during 2014-2015, are likely to cause serious consequences.

\section{Conclusions}

This study presents detailed analyses on the characteristics of reassortment of human infected AIV H5N6 currently circulated in China. It confirmed that AIV H5N6 isolates from both human and poultry in China during 2014-2015 were very heterogeneous; not only H5N1, but also $\mathrm{H} 9 \mathrm{~N} 2$, were involved in the reassortment of AIV H5N6. Also, both these AIV H5N6 isolates from human and poultry and their reassortment donors had experienced complicated evolutionary history. Therefore, the surveillance for influenza itself and its hosts from the perspective of epidemiology, virology and ecology should be further strengthened, especially on AIVs H9N2 and H5N1. 


\section{Additional files}

Additional file 1: 1a-8a: Matrices of genomes involved in this study. 1a, PB2 (fas 4.73mb); 2a, PB1 (fas 4.72mb); 3a, PA (fas 4.45mb); 4a, HA (fas $1.88 \mathrm{mb}$ ); 5a, NP (fas 3.26mb); 6a, NA (fas 1.62mb); 7a, MP (fas 2.33mb); 8a, NEP (fas $1.99 \mathrm{mb})$. (ZIP $1 \mathrm{mb}$ )

Additional file 2: $1 \mathrm{~b}-8 \mathrm{~b}$ : Large-scale phylogenetic trees involved in this study. 1b, PB2 (mts 17.0mb); 2b, PB1 (mts 16.8mb); 3b, PA (mts 16.7mb); 4b, HA (mts 4.90mb); 5b, NP (mts $17.5 \mathrm{mb}$ ); 6b, NA (mts 4.62mb); 7b, MP (mts 18.7mb); 8b, NEP (mts 17.3mb). (ZIP $18 \mathrm{mb}$ )

Additional file 3: $2 c, 3 c, 7 c$, and $8 c$ : Four accuracy phylogenetic trees involved in this study. 2c, PB1 (pdf 29.7 kb); 3c, PA (pdf 29.7 kb); 7c, MP (pdf $29.6 \mathrm{~kb}$ ); 8c, NEP (pdf $29.5 \mathrm{~kb}$ ). 1c (PB2), 4c (HA), 5c (NP), and 6c (NA) were put into the main text as Fig. 1 and Fig. 2. (ZIP 106 kb)

\section{Acknowledgements}

We thank all the scientists for having submitted the genomes of AIV H5N6 to NCBI Influenza Virus Sequence Database and the Global Initiative on Sharing Avian Influenza Data (GISAID) database.

\section{Funding}

This research was supported by grants from the Talent Programs for Fostering Outstanding Youth of Shanghai (grants number XYQ2013071), the National Science Fund for Distinguished Young Scholars (grants number 81325017), Chang Jiang Scholars Program (grants number T2014089), and the Key Discipline Construction of Public Health of Shanghai of Shanghai Municipal Health and Family Planning Commission (grants number 12GWZX0101).

\section{Availability of data and materials}

Data of the gene sequences used in this study can be obtain from two public databases, the NCBI Influenza Virus Sequence Database (http:// www.ncbi.nlm.nih.gov/genomes/FLU/aboutdatabase.html) and the Global Initiative on Sharing Avian Influenza Data (GISAID) database (http:// platform.gisaid.org/epi3/frontend).

The dataset supporting the conclusions of this article is available by request to the correspondence author (xiongchenglong@fudan.edu.cn, C. X.), who is the member of Influenza Research Group of the School of Public Health, Fudan University.

\section{Authors' contributions}

All authors made significant contributions to the conception, data acquisition, analysis and drafting of this manuscript and approve the final version submitted. CX, ZZ and QJ conceived and designed the project; CX, $\mathrm{ZZ}$ and $\mathrm{RL}$ developed the research question. CX and ZZ also contributed to the analysis design and interpretation of the data. GZ supervised the sequences collection. $L J$ and $C X$ collected the sequences and calculated them. YC managed the data from the study and assisted in language translation.

\section{Competing interests}

We declare that we have no competing interest. Although Dr. Zhijie Zhang is an Associate Editor of this journal, he was not involved in handling this manuscript.

\section{Consent for publication}

Not Applicable.

\section{Ethics approval and consent to participate}

This study is a serial of phylogenetic analyses based on large scale of existing gene sequences; all these sequences can be searched, BLASTed, and downloaded from two public databases, the NCBI Influenza Virus Sequence Database and the Global Initiative on Sharing Avian Influenza Data (GISAID) database. No institutional review board approval was required from the research ethics committee of School of Public Health, Fudan University, and animals' ethics approval was applicable neither.

\section{Author details}

'Department of Epidemiology and Biostatistics, School of Public Health, Fudan University, Shanghai, People's Republic of China. ${ }^{2}$ Key Laboratory of Public Health Safety, Ministry of Education, Fudan University, Shanghai, People's Republic of China. ${ }^{3}$ Department of Public Health Microbiology, School of Public Health, Fudan University, Shanghai, People's Republic of China. ${ }^{4}$ School of Epidemiology, Public Health and Preventive Medicine, Faculty of Medicine, University of Ottawa, Ottawa, ON, Canada. ${ }^{5}$ Bldg. 8\#, Rd. Dong'an 130, Shanghai 200032, People's Republic of China.

Received: 12 March 2016 Accepted: 14 October 2016 Published online: 25 October 2016

\section{References}

1. Liu D, Shi W, Shi Y, et al. Origin and diversity of novel avian influenza A H7N9 viruses causing human infection: phylogenetic, structural, and coalescent analyses. Lancet. 2013;381:1926-32.

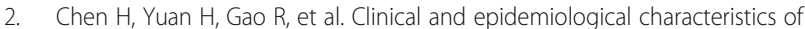
a fatal case of avian influenza A H10N8 virus infection: a descriptive study. Lancet. 2014;383:714-21.

3. Yang ZF, Mok CK, Peiris JS, Zhong NS. Human infection with a novel avian influenza a(H5N6) virus. N Engl J Med. 2015:373:487-9.

4. Zhuang PH, Lo W. Sichuan man dies in first human case of H5N6 bird flu. China daily news center media report. 2nd human H5N6 avian flu reported in S. China. 2014. http://www.chinadaily.com.cn/m/chinahealth/2016-01/05/ content_22940148.htm. Accessed 5 Jan 2016.

5. WHO. Disease outbreak news, human infection with avian influenza A(H5N6) virus-China. http://www.who.int/csr/don/26-january-2016-avianinfluenza-china/en/. Accessed 18 Feb 2016.

6. Chen T, Zhang R. Symptoms seem to be mild in children infected with avian influenza A (H5N6) and other subtypes. J Infect. 2015;71:702-3.

7. Mok CK, Da Guan W, Liu XQ, et al. Genetic characterization of highly pathogenic avian influenza a(H5N6) virus, Guangdong. China Emerg Infect Dis. 2015;21:2268-71.

8. Bi Y, Mei K, Shi W, et al. Two novel reassortants of avian influenza A (H5N6) virus in China. J Gen Virol. 2015;96:975-81.

9. Pan $M, G a o$ R, Lv Q, et al. Human infection with a novel, highly pathogenic avian influenza A (H5N6) virus: Virological and clinical findings. J Infect. 2016:72:52-9.

10. Ghedin E, Fitch A, Boyne A, et al. Mixed infection and the genesis of influenza virus diversity. J Virol. 2009;83:8832-41.

11. Dugan VG, Chen R, Spiro DJ, et al. The evolutionary genetics and emergence of avian influenza viruses in wild birds. PLoS Pathog. 2008; doi: 10.1371/journal.ppat.1000076.

12. Guan Y, Shortridge KF, Krauss S, Webster RG. Molecular characterization of H9N2 influenza viruses: were they the donors of the "internal" genes of H5N1 viruses in Hong Kong? Proc Natl Acad Sci U S A. 1999;96:9363-7.

13. Xu X, Cox N, Guo Y. Am. Soc. Virol. 17th Annu. Meeting. 1998. Vancouver, British Columbia, Canada, W29-5, 110

14. Guan $Y$, Smith GJ. The emergence and diversification of panzootic H5N1 influenza viruses. Virus Res. 2013;178:35-43.

15. Pu J, Wang S, Yin Y, et al. Evolution of the H9N2 influenza genotype that facilitated the genesis of the novel H7N9 virus. Proc Natl Acad Sci U S A. 2015:112:548-53.

16. Su S, Bi Y, Wong G, Gray GC, Gao GF, Li S. Epidemiology, Evolution, and Recent Outbreaks of Avian Influenza Virus in China. J Virol. 2015;89:8671-6.

17. Lin YP, Shaw M, Gregory $V$, et al. Avian-to-human transmission of H9N2 subtype influenza A viruses: relationship between H9N2 and H5N1 human isolates. Proc Natl Acad Sci U S A. 2000;97:9654-8.

18. Cheng X, Liu J, He J, Shan F. Virological and serological surveys for H9N2 subtype of influenza A virus in chickens and men in Shenzhen city. Zhonghua Shi Yan He Lin Chuang Bing Du Xue Za Zhi. 2002;16:319-21 (in Chinese).

19. Shi W, Li W, Li X, et al. Phylogenetics of varied subtypes of avian influenza viruses in China: potential threat to humans. Protein Cell. 2014;5:253-7.

20. Wu Y, Gao GF. Lessons learnt from the human infections of avian-origin influenza A H7N9 virus: live free markets and human health. Sci China Life Sci. 2013:56:493-4

21. Kawaoka Y, Webster RG. Sequence requirements for cleavage activation of influenza virus hemagglutinin expressed in mammalian cells. Proc Natl Acad Sci U S A. 1988;85:324-8. 22. 
22. Li S, Liu C, Klimov A, Subbarao K, et al. Recombinant influenza A virus vaccines for the pathogenic human A/Hong Kong/97 (H5N1) viruses. J Infect Dis. 1999;179:1132-8.

23. Hu T, Song J, Zhang W, et al. Emergence of novel clade 2.3.4 influenza A (H5N1) virus subgroups in Yunnan Province, China. Infect Genet Evol. 2015; 33:95-100.

24. Wong FY, Phommachanh P, Kalpravidh W, et al. Reassortant highly pathogenic influenza A(H5N6) virus in Laos. Emerg Infect Dis. 2015;21:511-6.

Submit your next manuscript to BioMed Central and we will help you at every step:

- We accept pre-submission inquiries

- Our selector tool helps you to find the most relevant journal

- We provide round the clock customer support

- Convenient online submission

- Thorough peer review

- Inclusion in PubMed and all major indexing services

- Maximum visibility for your research

Submit your manuscript at www.biomedcentral.com/submit 